\title{
A Multifactorial Histopathologic Score for the Prediction of Prognosis of Resected Esophageal Adenocarcinomas After Neoadjuvant Chemotherapy
}

\author{
Rupert Langer, $\mathrm{MD}^{1,2}$, Karen Becker, $\mathrm{MD}^{2}$, Inti Zlobec, $\mathrm{PhD}^{1}$, Ralf Gertler, $\mathrm{MD}^{3}$, Leila Sisic, $\mathbf{M D}^{4}$, \\ Markus Büchler, $\mathrm{MD}^{4}$, Florian Lordick, $\mathrm{MD}^{5}$, Julia Slotta-Huspenina, $\mathrm{MD}^{2}$, Wilko Weichert, $\mathrm{MD}^{6}$, \\ Heinz Höfler, $\mathrm{MD}^{2}$, Marcus Feith, $\mathrm{MD}^{3}$, and Katja Ott, $\mathrm{MD}^{4}$ \\ ${ }^{1}$ Institute of Pathology, University of Bern, Bern, Switzerland; ${ }^{2}$ Institute of Pathology, Technische Universität München, \\ Munich, Germany; ${ }^{3}$ Department of Surgery, Klinikum Rechts der Isar, Technische Universität München, Munich, \\ Germany; ${ }^{4}$ Department of Surgery, University of Heidelberg, Heidelberg, Germany; ${ }^{5}$ University Cancer Center (UCCL) \\ at the University Clinic Leipzig, University of Leipzig, Leipzig, Germany; ${ }^{6}$ Institute of Pathology, University of \\ Heidelberg, Heidelberg, Germany
}

\begin{abstract}
Background. For esophageal adenocarcinoma treated with neoadjuvant chemotherapy, postoperative staging classifications initially developed for non-pretreated tumors may not accurately predict prognosis. We tested whether a multifactorial TNM-based histopathologic prognostic score (PRSC), which additionally applies to tumor regression, may improve estimation of prognosis compared with the current Union for International Cancer Control/American Joint Committee on Cancer (UICC) staging system.

Patients and Methods. We evaluated esophageal adenocarcinoma specimens following cis/oxaliplatin-based therapy from two separate centers (center 1: $n=280$; and center 2: $n=80$ ). For the PRSC, each factor was assigned a value from 1 to 2 (ypT0-2 = 1 point; ypT3-4=2 points;
\end{abstract}

Electronic supplementary material The online version of this article (doi:10.1245/s10434-013-3410-y) contains supplementary material, which is available to authorized users.

Ruper Langer and Karen Becker contributed equally to this work as first authors.

Marcus Feith and Katja Ott contributed equally to this work as last authors.

(C) Society of Surgical Oncology 2013

First Received: 20 September 2013;

Published Online: 27 November 2013

R. Langer, MD

e-mail: rupert.langer@pathology.unibe.ch
ypN0 $=1$ point; $\quad$ ypN1-3 $=2$ points; $\leq 50 \%$ residual tumor/tumor bed $=1$ point; $>50 \%$ residual tumor/tumor bed $=2$ points). The three-tiered PRSC was based on the sum value of these factors (group A: 3; group B: 4-5; group $C: 6$ ) and was correlated with patients' overall survival (OS).

Results. The PRSC groups showed significant differences with respect to OS $(p<0.0001$; hazard ratio [HR] 2.2 [95\% CI 1.7-2.8]), which could also be demonstrated in both cohorts separately (center $1 p<0.0001$; HR 2.48 [95\% CI 1.8-3.3] and center $2 p=0.015$; HR 1.7 [95\% CI 1.1-2.6]). Moreover, the PRSC showed a more accurate prognostic discrimination than the current UICC staging system ( $p<0.0001$; HR 1.15 [95\% CI 1.1-1.2]), and assessment of two goodness-of-fit criteria (Akaike Information Criterion and Schwarz Bayesian Information Criterion) clearly supported the superiority of PRSC over the UICC staging.

Conclusion. The proposed PRSC clearly identifies three subgroups with different outcomes and may be more helpful for guiding further therapeutic decisions than the UICC staging system.

Preoperative chemo- or radiochemotherapy (CTX or RCTX) followed by resection has been shown to provide survival benefit for patients with locally advanced esophageal adenocarcinoma compared with surgery alone ${ }^{1-4}$. Accurate postoperative staging is important for correct estimation of prognosis and for further therapeutic decisions. The new Union for International Cancer Control/ 
American Joint Committee on Cancer (UICC/AJCC) TNM classification (7th edition; TNM7) shows a better prognostic stratification for primary resected esophageal cancer compared with the 6th edition (TNM6) ${ }^{5,6}$. However, the accuracy of this current classification in neoadjuvant-treated esophageal adenocarcinoma is unknown ${ }^{7}$. For gastric cancer, we have recently demonstrated that a multifactorial histopathologic prognostic score (PRSC) that included the factors ypT category, ypN category and degree of histopathological tumor regression ${ }^{8}$ could accurately classify three groups of patients with different outcomes after neoadjuvant CTX followed by surgery ${ }^{9}$. In this study we investigated whether a similar, but tumor site-specific, PRSC can be applied in esophageal adenocarcinoma. Moreover, we aimed to determine whether this PRSC may provide a more accurate estimation of the prognosis compared with the current UICC/AJCC staging system.

\section{MATERIALS AND METHODS}

\section{Patients}

Three hundred and sixty resection specimens from patients with histologically confirmed, locally advanced esophageal adenocarcinoma [staged cT3/4 N(any) cM0] from two independent academic centers were investigated. There were 14 females $(3.9 \%)$ and 346 males (96.1\%), with a median age of 57 years (range $25-80$ years). The median survival, which was calculated from the day of surgery onward, was 37 months (95\% confidence interval [CI] 28-45 months). There was no difference between both centers regarding age, gender distribution, or survival. Patients had undergone neoadjuvant cis/oxaliplatin/5FUbased CTX (mainly cisplatin/5FU/leucovorin regimen) followed by esophagectomy without adjuvant treatment between 1995 and 2010 at the Department of Surgery at the Technische Universität München $(n=280)$ or the University of Heidelberg $(n=80)^{10}$. Eighteen patients in the Munich cohort and 13 patients in the Heidelberg cohort (total 31 patients) had been treated with additional radiotherapy. The protocols for neoadjuvant treatment had been approved by the Institutional Review Boards. Table 1 summarizes tumor and patient characteristics, including treatment.

\section{Histopathologic Evaluation}

The resection specimens had been prospectively examined according to a standardized protocol ${ }^{8,11}$ which included the investigation of the entire macroscopically identifiable tumor or the area with scarring indicating the
TABLE 1 Tumor and patient characteristics of the two patient cohorts

\begin{tabular}{|c|c|c|c|}
\hline Factor & $\begin{array}{l}\text { Center 1 } \\
\text { (Munich) }\end{array}$ & $\begin{array}{l}\text { Center } 2 \\
\text { (Heidelberg) }\end{array}$ & Total \\
\hline \multicolumn{4}{|l|}{ ypT category } \\
\hline урТ0 & 21 & 17 & 38 \\
\hline ypT1 & 39 & 4 & 43 \\
\hline урT2 & 51 & 13 & 64 \\
\hline урT3 & 165 & 42 & 207 \\
\hline урТ4 & 4 & 4 & 8 \\
\hline \multicolumn{4}{|l|}{ ypN category } \\
\hline ypN0 & 121 & 38 & 159 \\
\hline ypN1 & 52 & 20 & 72 \\
\hline ypN2 & 57 & 7 & 64 \\
\hline ypN3 & 50 & 15 & 65 \\
\hline \multicolumn{4}{|l|}{ Distant metastases } \\
\hline Absent & 235 & 64 & 299 \\
\hline Present & 45 & 16 & 61 \\
\hline \multicolumn{4}{|l|}{ Tumor grading } \\
\hline G1 & 2 & 0 & 2 \\
\hline $\mathrm{G} 2$ & 94 & 29 & 123 \\
\hline G3 & 149 & 49 & 198 \\
\hline G4 & 10 & 0 & 10 \\
\hline \multicolumn{4}{|l|}{$\mathrm{R}$ category } \\
\hline R0 & 231 & 80 & 311 \\
\hline $\mathrm{R} 1$ & 43 & 0 & 43 \\
\hline $\mathrm{R} 2$ & 6 & 0 & 6 \\
\hline \multicolumn{4}{|c|}{ Tumor regression grade (Becker) } \\
\hline TRG1a & 21 & 17 & 38 \\
\hline TRG1b & 59 & 8 & 64 \\
\hline TRG2 & 63 & 11 & 77 \\
\hline TRG3 & 137 & 44 & 181 \\
\hline \multicolumn{4}{|l|}{ Neoadjuvant treatment } \\
\hline PLF & 167 & 9 & 176 \\
\hline PLF-Taxol & 65 & 1 & 66 \\
\hline OLF & 22 & 3 & 25 \\
\hline EOX & 2 & 48 & 50 \\
\hline RCTX & 18 & 13 & 31 \\
\hline $\begin{array}{l}\text { Other platin-based } \\
\text { CTX }\end{array}$ & 6 & 6 & 12 \\
\hline Total & 280 & 80 & 360 \\
\hline
\end{tabular}

$P L F$ cisplatin/5FU/leucovorine, $O L F$ oxaliplatin/5FU/leucovorin, $E O X$ epirubicin, oxaliplatin, capecitabin, $R C T X$ radiochemotherapy ( $\geq 45$ Gy cis/oxaliplatin based)

previous site of the tumor (the tumor bed). Tumor regression grading (TRG; according to Becker et al. ${ }^{8}$ ) was based on an estimation of the percentage of residual tumor tissue in relation to the macroscopically identifiable tumor bed at the primary site of the tumor, consisting of three grades: grade 1 -complete or subtotal regression $(<10 \%$ residual 
FIG. 1 Histopathologic prognostic score (PRSC): a construction of PRSC; b PRSC and 5-year overall survival a

\begin{tabular}{|c|c|c|}
\hline & \multicolumn{2}{|c|}{ value } \\
\hline Parameter & 1 point & 2 points \\
\hline УPT category & урТО-2 & уртз-4 \\
\hline ypN category & ypNo & ypN1-3 \\
\hline tumor regression grade & $\begin{array}{l}\text { TRG1a; TRG1b; TRG2 } \\
\text { (<50\% residual tumor) }\end{array}$ & $\begin{array}{l}\text { TRG3 } \\
\text { (250\% residual tumor) }\end{array}$ \\
\hline \multicolumn{3}{|c|}{ 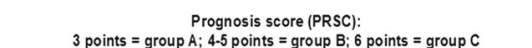 } \\
\hline
\end{tabular}

PRSC

- Group A

Group B

+++ Censored b

Estimated probability

of survival $\quad \mathrm{P}<0.001$

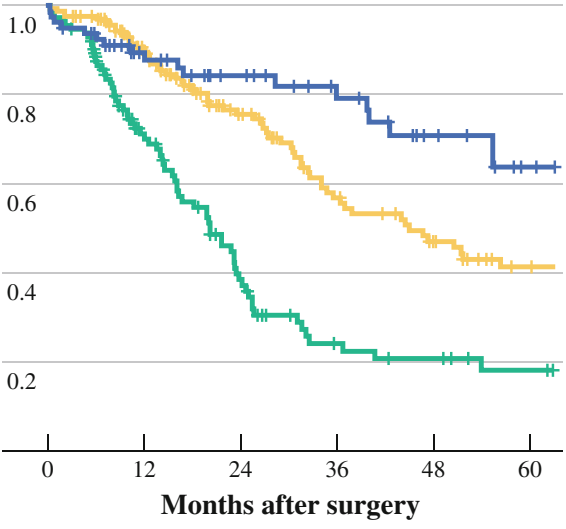

tumor per tumor bed; grade 1a is complete regression and grade $1 \mathrm{~b}$ is subtotal regression); grade 2-partial tumor regression (10-50\% residual tumor per tumor bed); and grade 3 - minimal or no tumor regression ( $>50 \%$ residual tumor per tumor bed). All cases were reclassified according to the current UICC/AJCC TNM system ${ }^{12}$.

\section{Prognostic Score}

Analogous to our previous study in gastric cancer, ${ }^{9}$ the factors TRG, ypT and ypN category were first each assigned a point value according to the respective prognostic impact (see electronic supplementary material [ESM] file 1): TRG (TRG factor): grade 1 and grade 2 (i.e. $<50 \%$ residual tumor) $=1$ point; grade 3 (i.e. $\geq 50 \%$ residual tumor) $=2$ points; UICC/AJCC ypT category (ypT factor): ypT0-ypT2 = 1 point; урT3-ypT4 = 2 points; UICC/AJCC ypN category (ypN factor): ypN0 $=1$ point; ypN1-ypN3 $=2$ points. The raw PRSC consisted of the sum of the values of these single factors with a possible range from 3 to 6 points, and was further subclassified into three groups of patients according to the survival curves of each sum score: Group A with a sum of 3 points, group B with a sum of 4-5 points, and group $C$ with a sum of 6 points (Fig. 1; see also ESM file 2).

\section{Statistical Analysis}

IBM SPSS statistics 21 software (SPSS Inc., Chicago, IL, USA) and SAS V9.2 (The SAS Institute, Cary, NC, USA)were used for statistics. Descriptive associations between single variables were evaluated by Chi squared tests and Fisher's exact tests. Univariate analysis of survival was performed using the Kaplan-Meier method to estimate survival probabilities in patient subgroups, and the log-rank test was used for statistical comparisons. Cox proportional hazard models were performed to investigate
TABLE 2 PRSC and UICC/AJCC stages

\begin{tabular}{cllllll}
\hline & \multicolumn{3}{l}{ UICC/AJCC stage } & Total \\
\cline { 2 - 5 } & $0^{\mathrm{a}}$ & I & II & III & IV & \\
\hline PRSC & & & & & & \\
A & 28 & 47 & 0 & 0 & 5 & 80 \\
B & 0 & 21 & 69 & 51 & 26 & 167 \\
C & 0 & 0 & 0 & 83 & 30 & 113 \\
Total & 28 & 68 & 69 & 134 & 61 & 360 \\
\hline
\end{tabular}

UICC/AJCC Union for International Cancer Control/American Joint Committee on Cancer, PRSC histopathologic prognostic score

${ }^{\text {a }}$ No UICC/AJCC stage for ypT0yN0

multivariate relationships of covariates with survival. Ninety-five percent CIs were used to determine the effect of each variable on outcome. All tests were two-sided, and the significance level was set at $5 \%$. In order to estimate the goodness-of-fit of each PRGS model in comparison to UICC/AJCC staging, the Akaike Information Criterion (AIC) and Schwarz Bayesian Information Criterion (SBC) were used. Both methods adjust the $-2 \log$ likelihood statistics for the number of parameters in the model and number of observations used. Lower values of AIC and SBC indicate superior model fit with the 'best' model showing the lowest values for both.

\section{RESULTS}

\section{Pathologic Findings}

The histopathologic findings of the tumors are given in Table 2. The survival curves, demonstrating the prognostic relevance of the value assignment of each single factor, are provided in the ESM supplemental file 1. No significant survival difference was found between the two patient groups of the two different surgical centers with respect to the respective factor subclassifications. 
Regarding single TNM parameters and tumor regression, a significant association was noted between tumor regression and the UICC ypT and ypN categories (each $p<0.001)$ in the total patient cohort of both centers. However, of the 66 cases with a subtotal tumor regression, $20(30.3 \%)$ had an infiltration of the adventitial tissue and beyond, and were therefore classified as ypT3 (19 cases, Fig. 1) and ypT4 (one case). Five of the 38 patients $(13.2 \%)$ with complete regression of the primary tumor had lymph node metastases, and five (13.2 \%) had distant metastases.

According to the UICC/AJCC anatomic staging system, 20 patients $(5.6 \%)$ had UICC/AJCC tumor stage IA, 48 patients $(13.3 \%)$ had stage IB, 52 patients $(14.4 \%)$ had stage IIA, 17 patients $(4.7 \%)$ had stage IIB, including three patients with complete tumor regression at the primary site of the tumor but with ypN1. Of 51 patients (14.2\%) with stage IIIA tumors, one patient had complete tumor regression at the primary site and but ypN2. Thirtyseven patients $(10.3 \%)$ were stage IIIB, 46 patients $(12.8 \%)$ were stage IIIC and 61 patients $(16.9 \%)$ had stage IV tumors, among them five patients with complete tumor regression. Twenty-eight patients $(7.8 \%)$ had no residual tumor at the primary site and no lymph node metastases. No difference was found between the two centers regarding the distribution of UICC/AJCC stages.

\section{Prognostic Score}

The PRSC classified three groups of patients: group A with 80 patients $(22.2 \%$ ), group B with 167 patients $(46.4 \%)$, and group C with 113 patients $(31.4 \%)$. In the Munich cohort, group A comprised 60 patients $(21.4 \%)$, group B comprised 137 patients (48.9\%), and group C comprised 83 patients $(29.6 \%)$. In the Heidelberg cohort 20 patients $(25 \%)$ were classified into group A, and 30 patients $(37.5 \%)$ each into group B and group C. No significant difference was noted between both centers regarding the distribution of prognostic groups.

\section{Comparison with Prognosis}

The PRSC showed a highly significant association with prognosis in the whole case collection $(p<0.0001)$, Munich collective $(p<0.0001)$, and the Heidelberg collective $(p=0.015)$. Regarding the whole case collection, the PRSC discriminated significantly between the three prognostic groups. In group A, the median overall survival (OS) was not reached. Group B patients had a median OS of 45 months (95\% CI 31-58 months), and group C patients had a median OS of 20 months (95\% CI 1426 months). The overall difference was highly significant
( $p<0.001, \log$ rank analysis), as were the differences between groups A and B $(p=0.029)$, groups A and C $(p<0.001)$ and groups $\mathrm{B}$ and $\mathrm{C}(p<0.001$; Fig. 1$)$. The PRSC also showed a significant prognostic value when separately analyzing the completely resected tumors without distant metastases $(p<0.001)$, the homogenous group of patients with PLF treatment $(p<0.001)$, the heterogenous group with other platin-based treatment $(p<0.001)$, and the small group of patients who were treated with RCTX ( $p=0.023)$.

In a multivariate analysis including the factors PRSC, tumor differentiation (grading), resection category, and the presence or absence of distant metastases at the time of surgery, the PRSC $(p<0.001$; hazard ratio [HR] 1.93; $95 \%$ CI 1.44-2.60) and tumor grading ( $p=0.006$; HR 1.54; $95 \%$ CI 1.31-2.09) were independent prognostic factors. The PRSC was still an independent prognostic factor when analyzing both cohorts separately (data not shown).

\section{Union for International Cancer Control/American Joint Committee on Cancer (UICC/AJCC) Staging}

There was a strong association between the UICC/AJCC anatomic staging system and the PRSC $(p=0.001)$. All patients with UICC/AJCC stages III and higher were in the unfavorable PRSC group C. However, there were 16 patients in the PRSC group A who had a UICC/AJCC II stage, and nine patients who would be classified as UICC III or IV. On the other hand, 21 patients in the PRSC group B were classified as UICC/AJCC stage I, and 77 patients were classified into the prognostically unfavorable UICC/ AJCC stages III/IV (Table 2). UICC/AJCC staging was prognostically relevant for stages 0 , I, II, III, and IV ( $p<0.001$; see Fig. 2a) and also when probing for the anatomic substages 0 , IA, IB, IIA, IIB, IIIA, IIIB, IIIC, and IV (see Fig. 2b). However, patients with anatomic stage IA had an outcome similar to those with stage IIa, whereas stage IB patients had the best clinical outcome, which was even better than patients with complete tumor regression and without lymph node metastases (ypTONO). A multivariate analysis including the factors UICC/AJCC staging, tumor regression, resection category, and tumor grading (differentiation) showed tumor regression $(p=0.005$; HR 1.34 ; $95 \%$ CI 1.09-1.65), tumor grading ( $p=0.003$; HR 1.66 ; $95 \%$ CI 1.18-2.33), and resection status ( $p=0.017$; HR 1.58; $95 \%$ CI 1.09-2.3) as independent prognostic factors, in contrast to UICC/AJCC staging ( $p=0.058$; HR 1.08; $95 \%$ CI 0.99-1.17).

Moreover, the comparison between the PRSC and the UICC staging groups showed significant advantages for the PRSC with higher hazard ratios and lower AIC and SBC 
FIG. 2 UICC/AJCC anatomic staging: a UICC/AJCC anatomic staging and 5year overall survival; b UICC/AJCC anatomic staging with subgroups and 5year overall survival. UICC/AJCC Union for International Cancer Control/ American Joint Committee on Cancer

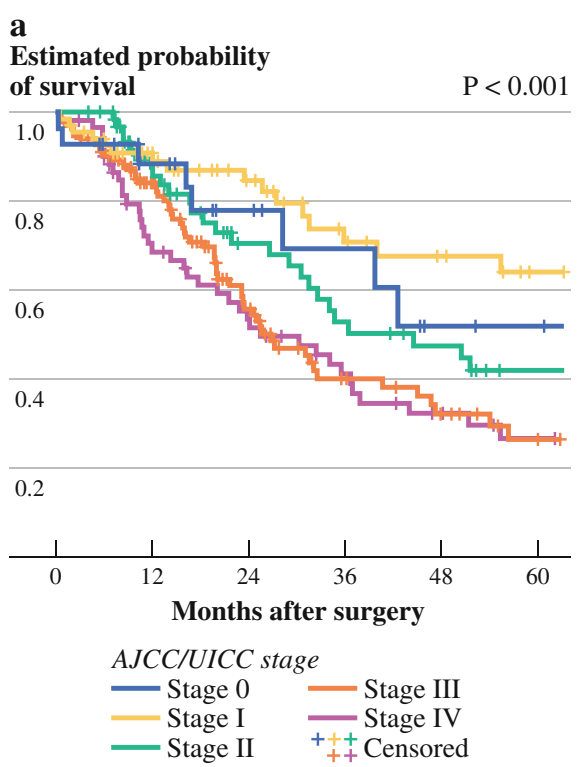

\section{b}

Estimated probability

of survival $\quad \mathrm{P}<0.001$

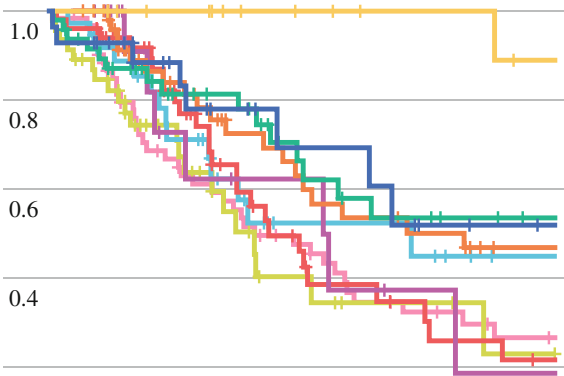

0.2

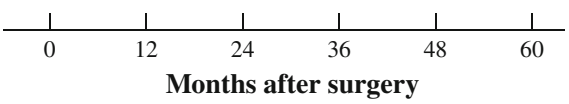

AJCC/UICC stage

- ypT0ypN0 - Stage IIA - Stage IIIC

- Stage IA Stage IIB Stage IV

Stage IB $\quad$ Stage IIIA +++ Censored
TABLE 3 Comparison of various goodness-of-fit criteria and tests of significance

\begin{tabular}{|c|c|c|c|}
\hline & PRSC & $\begin{array}{l}\text { UICC } \\
\text { (with subgroups) }\end{array}$ & $\begin{array}{l}\text { UICC } \\
(0, \text { I, II, III, IV })\end{array}$ \\
\hline \multicolumn{4}{|l|}{ Total collective } \\
\hline$p$ Value & $<0.0001$ & $<0.0001$ & 0.0065 \\
\hline HR $(95 \%$ CI $)$ & $2.2(1.7-2.8)$ & $1.15(1.1-1.2)$ & $1.108(1.0-1.1)$ \\
\hline AIC & 1530.757 & 1553.758 & 1566.636 \\
\hline SBC & 1533.787 & 1556.789 & 1569.667 \\
\hline \multicolumn{4}{|l|}{ Munich } \\
\hline$p$ Value & $<0.0001$ & $<0.0001$ & 0.0015 \\
\hline HR $(95 \%$ CI $)$ & $2.48(1.8-3.3)$ & $1.2(1.1-1.3)$ & $1.1(1.0-1.2)$ \\
\hline AIC & 1108.319 & 1123.707 & 1137.93 \\
\hline SBC & 1111.081 & 1126.469 & 1140.692 \\
\hline \multicolumn{4}{|l|}{ Heidelberg } \\
\hline$p$ Value & 0.0154 & 0.5997 & 0.9291 \\
\hline HR $(95 \%$ CI $)$ & $1.7(1.1-2.6)$ & $1.03(0.9-1.2)$ & $1.0(0.9-1.1)$ \\
\hline AIC & 260.691 & 266.61 & 266.881 \\
\hline SBC & 262.275 & 268.194 & 268.464 \\
\hline
\end{tabular}

PRSC histopathologic prognostic score, UICC Union for International Cancer Control, $H R$ hazard ratio, AIC Akaike Information Criterion, $S B C$ Schwarz Bayesian Information Criterion

values compared with the UICC staging groups. This was also found when analyzing the subcohorts of the collective and when comparing the UICC stages grouped to stages I, II, III, and IV without subgroups (Table 3). Therefore, the PRSC can be regarded as a more desirable model for prognostication in this patient collection.

\section{DISCUSSION}

We demonstrated in two cohorts of neoadjuvantlytreated patients with esophageal adenocarcinoma that an easily applicable scoring system (PRSC), which includes the factors UICC/AJCC ypT category, ypN category, and the degree of histopathological tumor regression serves as a simple but highly useful post-treatment and postoperative classification system. The PRSC revealed an accurate correlation with survival, thereby discriminating three groups of patients with significantly different outcomes. The proposed PRSC also had better performance regarding the estimation of prognosis than the current UICC/AJCC staging system in our collective.

The prognosis of patients with locally advanced esophageal adenocarcinoma has improved over the last decades due to advances in surgical techniques, patient selection, and staging methods, ${ }^{13,14}$ and due to the now widely performed multimodal treatment with peri- or preoperative CTX or chemoradiotherapy ${ }^{1-4,15}$. Complete or subtotal tumor regression can be observed in up to $30 \%$ of patients after CTX, a finding that has a significant prognostic impact. Another $20 \%$ of patients show partial tumor regression after neoadjuvant treatment ${ }^{1,3,11}$. The impact of histopathologic tumor regression after neoadjuvant treatment may even exceed the prognostic impact of the depth of tumor invasion (i.e. ypT category) ${ }^{11,16,17}$. Classification of tumor regression grade (TRG), which represents one part of the PRSC, has been proven to be objective and reproducible ${ }^{18,19}$. We used the TRG system according to Becker, which is based on the estimation of the percentage 
of residual tumor ${ }^{8,11,18,20}$. Other authors use similar percentage-based steps of residual tumor to define different TRGs, and have also described the $50 \%$ cutoff for residual tumor as prognostically relevant ${ }^{21,22}$. These systems, as well as the Mandard classification, ${ }^{23}$ which is based on the estimation of the relation of fibrosis to vital tumor, could be easily applied for the proposed PRSC since the relevant categories can be used in parallel to our system. The second important parameter, which heavily influences patient's outcome, is the presence of lymph node metas$\operatorname{tases}^{11,22,24}$. Although tumor differentiation was also an independent prognostic factor in our study, and is also implemented by the AJCC into the prognostic staging of early stages of untreated tumors, ${ }^{25,26}$ we did not include this factor in our PRSC. There may be considerable differences between the determination of tumor grading in preoperative biopsies and the corresponding resection specimen ${ }^{27}$. Furthermore, in the context of a multimodal setting, it has to be emphasized that the estimation of tumor differentiation in CTX- or RCTX-treated tumors in posttreatment specimens may not be representative of the tumor due to the marked therapy-induced cytotoxic changes (e.g. regression, cytopathic effects and high-grade cellular atypia ${ }^{8,28}$ ). Moreover, in single cases, preoperative biopsy material may be scarce and only contain superficial, highly altered cellular material that may be sufficient for a malignant diagnosis but not for accurate estimation of tumor differentiation. The recently updated UICC/AJCC TNM system (TNM7) ${ }^{12}$ recognizes esophageal adenocarcinoma as a separate tumor entity in contrast to esophageal squamous cell carcinoma. Several changes, compared with the previous UICC/AJCC TNM classification (TNM6), ${ }^{29}$ resulted in additional prognostic information ${ }^{5,26,30-32}$. Although advantages of the updated UICC/AJCC TNM system could also be demonstrated for patients undergoing neoadjuvant treatment, ${ }^{24,33}$ staging systems that have been originally developed for untreated, primary resected tumors may have limitations because they do not consider regressive alterations of the tumors ${ }^{16}$. Regarding the previous TNM6 UICC/AJCC staging system, several publications have addressed this issue and have proposed alternatives or modifications to the UICC/AJCC staging system $^{16,22,34}$. In our collectives, there were marked limitations, particularly in the lower UICC/AJCC stages, regarding the prognostic value of the proposed staging categories. Stage IA patients had the best outcome, whereas stages II-IV showed similar overall survival curves. Moreover, no explicit staging is provided for tumors that show a complete regression of the primary site of the tumor and absence of lymph node metastases. These ypT0ypN0 tumors had a slightly worse outcome compared with stage IA tumors. By contrast, classification of tumors that show complete regression of the primary tumor but not of lymph node or distant metastases, which was observed in ten cases, should result in a classification of stage IIB or higher. Unfortunately, the number of cases with this finding was too low to achieve reliable knowledge concerning the biological significance of vital metastases in cases with complete tumor regression of the primary tumor site. However, our results clearly show the limitations of the UICC/AJCC staging system to accurately discriminate prognostically relevant groups in our large cohort of esophageal adenocarcinoma patients.

\section{CONCLUSIONS}

The easily applicable PRSC revealed an accurate correlation with survival and outperformed the current UICC staging system. Because a similar score is also applicable in gastric cancer, ${ }^{9}$ such prognostically relevant, posttreatment and postoperative classification systems may be considered for future clinical practice in tailoring the treatment of patients with locally advanced adenocarcinomas of the upper gastrointestinal tract after neoadjuvant treatment.

DISCLOSURE The authors declare no conflicts of interest.

\section{REFERENCES}

1. Cunningham D, Allum WH, Stenning SP, Thompson JN, Van de Velde CJ, Nicolson M, et al. Perioperative chemotherapy versus surgery alone for resectable gastroesophageal cancer. $N$ Engl $J$ Med. 2006;355(1):11-20.

2. Siewert JR, Lordick F, Ott K, Stein HJ, Weber WA, Becker K, et al. Induction chemotherapy in Barrett cancer: influence on surgical risk and outcome. Ann Surg. 2007;246(4):624-8, discussion 8-31.

3. van Hagen P, Hulshof MC, van Lanschot JJ, Steyerberg EW, van Berge Henegouwen MI, Wijnhoven BP, et al. Preoperative chemoradiotherapy for esophageal or junctional cancer. $N$ Engl $J$ Med. 2012;366(22):2074-84.

4. Ychou M, Boige V, Pignon J-P, Conroy T, Bouché O, Lebreton $\mathrm{G}$, et al. Perioperative chemotherapy compared with surgery alone for resectable gastroesophageal adenocarcinoma: an FNCLCC and FFCD multicenter phase III trial. J Clin Oncol. 2011;29(13):1715-21.

5. Hsu PK, Wu YC, Chou TY, Huang CS, Hsu WH. Comparison of the 6th and 7th editions of the American Joint Committee on Cancer tumor-node-metastasis staging system in patients with resected esophageal carcinoma. Ann Thorac Surg. 2010;89(4):1024-31.

6. Talsma K, van Hagen P, Grotenhuis BA, Steyerberg EW, Tilanus HW, van Lanschot JJ, et al. Comparison of the 6th and 7th Editions of the UICC-AJCC TNM classification for esophageal cancer. Ann Surg Oncol. 2012;19(7):2142-8.

7. Gaur P, Hofstetter WL, Bekele BN, Correa AM, Mehran RJ, Rice DC, et al. Comparison between established and the worldwide esophageal cancer collaboration staging systems. Ann Thorac Surg. 2010;89(6):1797-803, 1804.e1-3. 
8. Becker K, Mueller JD, Schulmacher C, Ott K, Fink U, Busch R, et al. Histomorphology and grading of regression in gastric carcinoma treated with neoadjuvant chemotherapy. Cancer. 2003; 98(7):1521-30.

9. Becker K, Reim D, Novotny A, Zum Büschenfelde CM, Engel J, Friess H, et al. Proposal for a multifactorial prognostic score that accurately classifies 3 groups of gastric carcinoma patients with different outcomes after neoadjuvant chemotherapy and surgery. Ann Surg. 2012;256(6):1002-7.

10. Lordick F, Ott K, Krause BJ, Weber WA, Becker K, Stein HJ, et al. PET to assess early metabolic response and to guide treatment of adenocarcinoma of the oesophagogastric junction: the MUNICON phase II trial. Lancet Oncol. 2007;8(9):797-805.

11. Langer R, Ott K, Feith M, Lordick F, Siewert JR, Becker K. Prognostic significance of histopathological tumor regression after neoadjuvant chemotherapy in esophageal adenocarcinomas. Mod Pathol. 2009;22(12):1555-63.

12. Sobin L, Gospodarowicz M, Wittekind C. TNM classification of malignant tumours. New York: John Wiley \& Sons; 2010.

13. Ott K, Bader FG, Lordick F, Feith M, Bartels H, Siewert JR. Surgical factors influence the outcome after Ivor-Lewis esophagectomy with intrathoracic anastomosis for adenocarcinoma of the esophagogastric junction: a consecutive series of 240 patients at an experienced center. Ann Surg Oncol. 2009;16(4):1017-25.

14. Portale G, Hagen JA, Peters JH, Chan LS, DeMeester SR, Gandamihardja TA, et al. Modern 5-year survival of resectable esophageal adenocarcinoma: single institution experience with 263 patients. J Am Coll Surg. 2006;202(4):588-96, discussion 96-8.

15. Lordick F, Stein HJ, Peschel C, Siewert JR. Neoadjuvant therapy for oesophagogastric cancer. Br J Surg. 2004; 91(5):540-51.

16. Rizk NP, Venkatraman E, Bains MS, Park B, Flores R, Tang L, et al. American Joint Committee on Cancer staging system does not accurately predict survival in patients receiving multimodality therapy for esophageal adenocarcinoma. J Clin Oncol. 2007;25(5):507-12.

17. Brucher BL, Swisher SG, Konigsrainer A, Zieker D, Hartmann J, Stein $\mathrm{H}$, et al. Response to preoperative therapy in upper gastrointestinal cancers. Ann Surg Oncol. 2009;16(4):878-86.

18. Becker K, Langer R, Reim D, Novotny A, Meyer zum Buschenfelde C, Engel J, et al. Significance of histopathological tumor regression after neoadjuvant chemotherapy in gastric adenocarcinomas: a summary of 480 cases. Ann Surg. 2011;253(5):934-9.

19. Fareed KR, Ilyas M, Kaye PV, Soomro IN, Lobo DN, Parsons SL, et al. Tumour regression grade (TRG) analyses in patients with resectable gastro-oesophageal adenocarcinomas treated with platinum-based neoadjuvant chemotherapy. Histopathol. 2009;55(4):399-406.

20. Brucher BL, Stein HJ, Zimmermann F, Werner M, Sarbia M, Busch R, et al. Responders benefit from neoadjuvant radiochemotherapy in esophageal squamous cell carcinoma: results of a prospective phase-II trial. Eur J Surg Oncol. 2004;30(9):963-71.

21. Chirieac LR, Swisher SG, Ajani JA, Komaki RR, Correa AM, Morris JS, et al. Posttherapy pathologic stage predicts survival in patients with esophageal carcinoma receiving preoperative chemoradiation. Cancer. 2005;103(7):1347-55.

22. Schneider PM, Baldus SE, Metzger R, Kocher M, Bongartz R, Bollschweiler E, et al. Histomorphologic tumor regression and lymph node metastases determine prognosis following neoadjuvant radiochemotherapy for esophageal cancer: implications for response classification. Ann Surg. 2005;242(5):684-92.

23. Mandard AM, Dalibard F, Mandard JC, Marnay J, Henry-Amar $\mathrm{M}$, Petiot JF, et al. Pathologic assessment of tumor regression after preoperative chemoradiotherapy of esophageal carcinoma. Clinicopathologic correlations. Cancer. 1994;73(11):2680-6.

24. Mehta SP, Jose P, Mirza A, Pritchard SA, Hayden JD, Grabsch HI. Comparison of the prognostic value of the 6th and 7th editions of the Union for International Cancer Control TNM staging system in patients with lower esophageal cancer undergoing neoadjuvant chemotherapy followed by surgery. Dis Esophagus. 2012;26(2):182-8.

25. Ishwaran H, Blackstone EH, Apperson-Hansen C, Rice TW. A novel approach to cancer staging: application to esophageal cancer. Biostatistics. 2009;10(4):603-20.

26. Rice TW, Rusch VW, Ishwaran H, Blackstone EH, Worldwide Esophageal Cancer Collaboration. Cancer of the esophagus and esophagogastric junction: data-driven staging for the seventh edition of the American Joint Committee on Cancer/International Union Against Cancer Cancer Staging Manuals. Cancer. 2010; 16(16):3763-73.

27. Dikken JL, Coit DG, Klimstra DS, Rizk NP, van Grieken N, Ilson $\mathrm{D}$, et al. Prospective impact of tumor grade assessment in biopsies on tumor stage and prognostic grouping in gastroesophageal adenocarcinoma: relevance of the seventh edition American Joint Committee on Cancer Staging Manual revision. Cancer. 2012; 118(2):349-57.

28. Chang F, Deere H, Mahadeva U, George S. Histopathologic examination and reporting of esophageal carcinomas following preoperative neoadjuvant therapy: practical guidelines and current issues. Am J Clin Pathol. 2008;129(2):252-62.

29. Sobin L, Wittekind C, editors. UICC. TNM classification of malignant tumours. New York: Wiley; 2002.

30. Reid TD, Sanyaolu LN, Chan D, Williams GT, Lewis WG. Relative prognostic value of TNM7 vs TNM6 in staging oesophageal cancer. Br J Cancer. 2011;105(6):842-6.

31. Gertler R, Stein HJ, Langer R, Nettelmann M, Schuster T, Hoefler $\mathrm{H}$, et al. Long-term outcome of 2,920 patients with cancers of the esophagus and esophagogastric junction: evaluation of the New Union Internationale Contre le Cancer/American Joint Cancer Committee staging system. Ann Surg. 2011; 253(4):689-98.

32. Reeh M, Nentwich MF, von Loga K, Schade J, Uzunoglu FG, Koenig AM, et al. An attempt at validation of the Seventh edition of the classification by the International Union Against Cancer for esophageal carcinoma. Ann Thorac Surg. 2012;93(3):890-6.

33. Nomura M, Shitara K, Kodaira T, Hatooka S, Mizota A, Kondoh C, et al. Prognostic impact of the 6th and 7th American Joint Committee on Cancer TNM staging systems on esophageal cancer patients treated with chemoradiotherapy. Int $J$ Radiat Oncol Biol Phys. 2012;82(2):946-52.

34. Barbour AP, Jones M, Gonen M, Gotley DC, Thomas J, Thomson $\mathrm{DB}$, et al. Refining esophageal cancer staging after neoadjuvant therapy: importance of treatment response. Ann Surg Oncol. 2008;15(10):2894-902. 